\title{
CPTAC Treatments Codelist
}

National Cancer Institute

\section{Source}

National Cancer Institute. CPTAC Treatments Codelist. NCI Thesaurus. Code C158037.

Terminology used in support of the data collection efforts of the Clinical Proteomic

Tumor Analysis Consortium (CPTAC) with the focus on medical treatments. 